\title{
HISTORY AND MODES OF STAR FORMATION IN THE MOST ACTIVE REGION OF THE SMALL MAGELLANIC CLOUD, NGC 346*
}

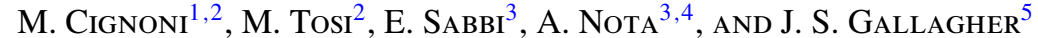 \\ ${ }^{1}$ Dipartimento di Astronomia, Università degli Studi di Bologna, via Ranzani 1, I-40127 Bologna, Italy \\ 2 Istituto Nazionale di Astrofisica, Osservatorio Astronomico di Bologna, Via Ranzani 1, I-40127 Bologna, Italy \\ ${ }^{3}$ Space Telescope Science Institute, 3700 San Martin Drive, Baltimore, MD, USA \\ ${ }^{4}$ European Space Agency, Research and Scientific Support Department, Baltimore, MD, USA \\ 5 Department of Astronomy, 475 North Charter Street, Madison, WI 53706, USA \\ Received 2010 June 5; accepted 2010 September 29; published 2010 December 29
}

\begin{abstract}
We discuss the star formation history of the Small Magellanic Cloud (SMC) region NGC 346 based on Hubble Space Telescope images. The region contains both field stars and cluster members. Using a classical synthetic color-magnitude diagram (CMD) procedure applied to the field around NGC 346 we find that the star formation pace there has been rising, from a quite low rate $13 \mathrm{Gyr}$ ago to $\approx 1.4 \times 10^{-8} M_{\odot} \mathrm{yr}^{-1} \mathrm{pc}^{-2}$ in the last $100 \mathrm{Myr}$. This value is significantly higher than that in other star-forming regions of the SMC. For NGC 346 itself, we compare theoretical and observed CMDs of several stellar sub-clusters identified in the region, and we derive their basic evolution parameters. We find that NGC 346 experienced different star formation regimes, including a dominant and focused "high-density mode," with the sub-clusters hosting both pre-main-sequence (PMS) and upper main-sequence (UMS) stars, and a diffuse "low-density mode," as indicated by the presence of low-mass PMS sub-clusters. Quantitatively, the star formation in the oldest sub-clusters started about $6 \mathrm{Myr}$ ago with remarkable synchronization, it continued at a high rate (up to $2 \times 10^{-5} M_{\odot} \mathrm{yr}^{-1} \mathrm{pc}^{-2}$ ) for about $3 \mathrm{Myr}$ and is now progressing at a lower rate. Interestingly, sub-clusters mainly composed of low-mass PMS stars now seem to experience the first episode of star formation, following multi-seeded spatial patterns, instead of resulting from a coherent trigger. Two speculative scenarios are put forth to explain the deficiency of UMS stars: the first invokes underthreshold conditions of the parent gas and the second speculates that the initial mass function is a function of time, with the youngest sub-clusters not having had sufficient time to form more massive stars.
\end{abstract}

Key words: galaxies: star clusters: general - Magellanic Clouds - stars: formation - stars: pre-main sequence

Online-only material: color figures

\section{INTRODUCTION}

The Small Magellanic Cloud (SMC) is the closest starforming galaxy with a low metallicity $(Z=0.004)$ typical of late-type dwarfs and most similar to that of primordial galaxies. For this reason, an increasing number of studies are being devoted to its star formation history (SFH) and related processes. This research is part of a long-term project aimed at studying how the star formation started and propagated in the SMC, studying both young clusters and the field population. We concentrate here on the OB association NGC 346, the most active star-forming region, where large numbers of pre-mainsequence (PMS) and massive stars are found. The inherent complexity of this star-forming cloud is well recognized. This region provides an excellent sample of newly formed stars spanning a wide range of masses and bridging a wide range of temporal and spatial scales.

The results presented in this paper are derived from observations acquired with the Advanced Camera for Survey (ACS) on board the Hubble Space Telescope (HST). Several photometric investigations tackled this complex population; all of them, using isochrone fitting, agreed that star formation has taken place in a variety of sub-clusters at different local conditions. How-

\footnotetext{
* Based on observations with the NASA/ESA Hubble Space Telescope, obtained at the Space Telescope Science Institute, which is operated by the Association of Universities for Research in Astronomy (AURA), Inc., under NASA contract NAS5-26555. These observations are associated with program GO10248.
}

ever, different formation scenarios are proposed: Sabbi et al. (2007) suggested a nearly coeval star formation in the cloud about 3 Myr ago. Contursi et al. (2000) proposed a progressive star formation from the central cluster and propagating along the Bar. Gouliermis et al. (2008) argued that NGC 346 has been shaped by two delayed triggering events: the first one due to the central cluster and the second one due to the massive progenitor of SNR B0057-724.

The purpose of this study is to (1) re-examine the star formation of the individual star-forming sites to a much finer spatial scale $(\sim 1 \mathrm{pc})$ by means of the synthetic color-magnitude diagram (CMD) approach and (2) evaluate systematically whether and how the initial mass function (IMF) and the star formation rate (SFR) are shaped and modified by the environment.

\section{STELLAR CONTENT AND ITS SPATIAL DISTRIBUTION IN THE REGION OF NGC 346}

Figure 1 shows our HST image of NGC 346, acquired with three overlapping pointings of the ACS Wide Field Channel (Nota et al. 2006). The analysis of this data set is presented in Sabbi et al. (2007) (hereafter S07). Assuming an intrinsic distance modulus to the SMC of $(m-M)_{0}=18.9$, the field of view covers about $88 \times 88 \mathrm{pc}^{2}$ and contains different stellar populations, both clustered and diffuse. The clustered population has two main components: the intermediate age cluster BS 90 (Bica \& Schmitt 1995), clearly recognizable as a roundish system at the top (north) of the image, and several clumps of stars ("sub-clusters" according to the definition 


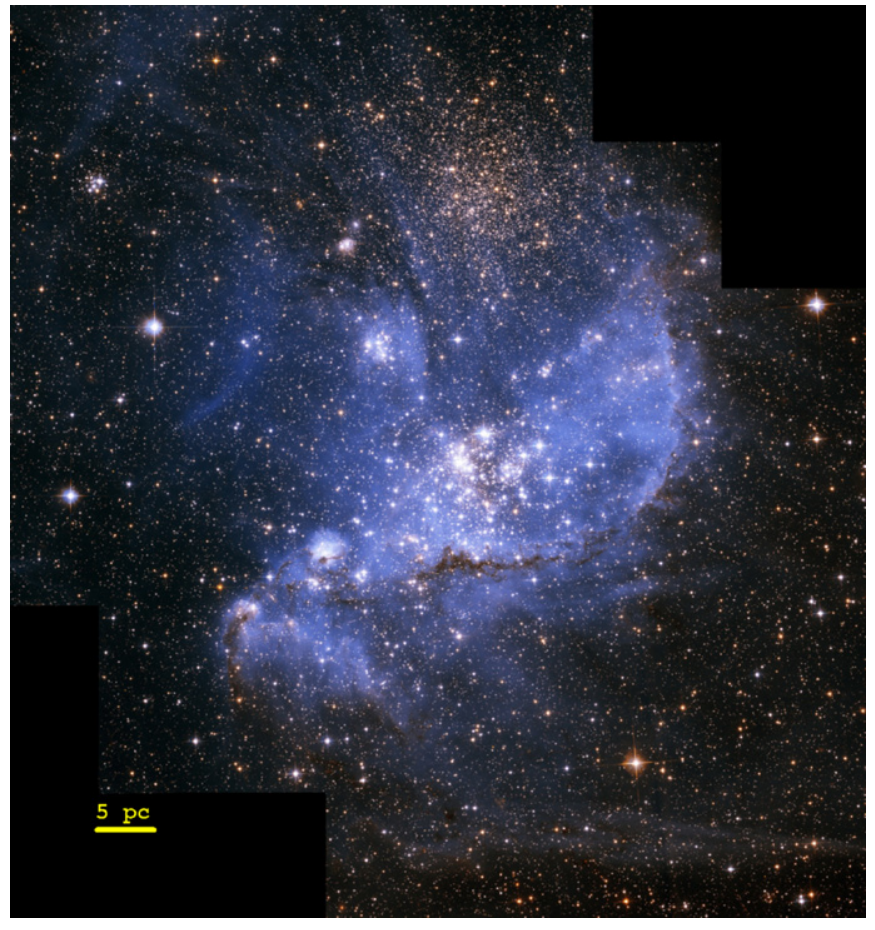

Figure 1. ACS image for the observed region around NGC 346 (Nota et al. 2006).

(A color version of this figure is available in the online journal.)

by S07), spread over the field along a sort of umbrella shape, and identified by S07. The diffuse population is uniformly spread over the field. BS 90 is 4-5 Gyr old and most likely located at the foreground of the NGC 346 cluster (see S07 and Cignoni et al. 2010). The sub-clusters are all part of the NGC 346 region, with ages from a few to $20 \mathrm{Myr}$ (S07; Gouliermis et al. 2008; Cignoni et al. 2010), while the diffuse population is presumably representative of the field SMC population in that region. The latter is then composed of SMC fore and background stars along the line of sight, possibly covering several kpc (see, e.g., Glatt et al. 2008) and spanning a wide range of ages, from Myr to $10 \mathrm{Gyr}$.

Our goal is to study the SFH of all these components by interpreting their observational CMDs with synthetic ones. BS 90 has already been studied by S07 (see also Rochau et al. 2007) and no further analysis is presented here. We thus concentrate on the other two components. The derivation of the $\mathrm{SFH}$ of the diffuse component is a standard application of the synthetic CMD method (see, e.g., Tosi et al. 1991; Cignoni et al. 2006; Cignoni \& Tosi 2010) to a statistically significant sample of field stars properly located in the region far from the subclusters. However, the analysis of the clustered component is handled with a dedicated methodology for two main reasons: (1) the various sub-clusters appear to have somewhat different ages and therefore we cannot treat them all together; and (2) each sub-cluster contains few stars and therefore, when treated individually, has rather large associated statistical uncertainties. So, for the sub-clusters we compared the observed CMDs with each other and with synthetic ones, taking into account the low number statistics and systematics.

Following the classification scheme proposed in Contursi et al. (2000), the NGC 346 region can be divided into a Spur, a filamentary low-density structure oriented to the northeast direction, and a fan-shaped feature (Bar), hosting the bulk of intermediate and massive stars.
The first question is whether two stellar tracers of the most recent activity, namely, objects on the upper main-sequence (UMS) and objects still on the PMS, are sharing the same "fine" spatial structure. A similar analysis has been performed by Schmeja et al. (2009) with the goal of deriving the clustering behavior, while the purpose here is to identify sub-clusters with peculiar mass function and to perform an accurate CMD analysis.

The main advantage of using two different tracers stems from their different observational and intrinsic uncertainties. With respect to UMS stars, PMS stars suffer less external contamination and low number statistics. However, UMS stars are less affected by incompleteness and theoretical uncertainties.

The regions of the CMD we associate with UMS and PMS stars are indicated in the top left panel of Figure 2 (with different colors in the online journal). For UMS stars (black open diamonds) we chose all objects above the Turn-On ( $\left.\mathrm{TOn}^{6}\right)$ of a $3 \mathrm{Myr}$ isochrone and bluer than $V-I=0.2$. With this definition, the UMS sample is mostly composed by intermediate mass stars and by a few massive stars. For the PMS stars we considered two samples: objects redder than the $3 \mathrm{Myr}$ isochrone with magnitude in the range $22.5<V<25$ (blue open circles, hereafter PMS sample) and fainter than $V=25$ (magenta open squares, low-mass PMS sample, hereafter, LPMS).

It is easy to notice that while the PMS sample is younger than $3 \mathrm{Myr}$ (or only slightly older if a modest additional reddening is taken into account), the UMS sample can include MS stars as old as 600 Myr. Moreover, while there is no doubt that the PMS and LPMS samples are free from any contamination, the UMS sample can include a minor fraction of PMS stars starting to approach the MS.

The top right, bottom right, and bottom left panels of Figure 2 show the location of the selected UMS, PMS, and LPMS stars, respectively. These distributions provide a clue to the history of the region: half of the UMS stars are clumped into a few agglomerates (SC-1, SC-13, and SC-16, named according to S07), while the other half are more evenly distributed and are probably members of the SMC field (foreground and background stars). One of the most striking aspects of this distribution is the absence of filamentary structures and a rather round appearance of the sub-clusters.

In contrast, the PMS stars are found almost exclusively either clumped or irregularly arranged along filaments. The distribution of the LPMS sample is also more filamentary than clumpy. In particular, we note that the Spur region, composed of a few aggregates in the PMS map, becomes a sort of bridge extending for tens of $\mathrm{pc}$ in the LPMS map. A word of caution is however necessary on the frequent holes in the star distribution: like that clearly visible in the center of SC-1, they are likely due to incompleteness effects. Incompleteness is caused by crowding, which reaches a maximum in the central region SC-1, and is much less severe at the outskirts of the region.

\section{SUB-CLUSTER PROPERTIES}

Looking at the maps of Figure 2, there is a further intriguing aspect of the star spatial distribution: for reasons that will be discussed later, not all the sub-clusters visible in the UMS map are detected in the PMS map and vice versa. In Figure 2, stellar sub-clusters which host both species are indicated with red arrows, while blue and green arrows indicate sub-clusters

\footnotetext{
6 The TOn is the point in the color-magnitude diagram where the PMS stars
} join the main sequence (see, e.g., Cignoni et al. 2010). 

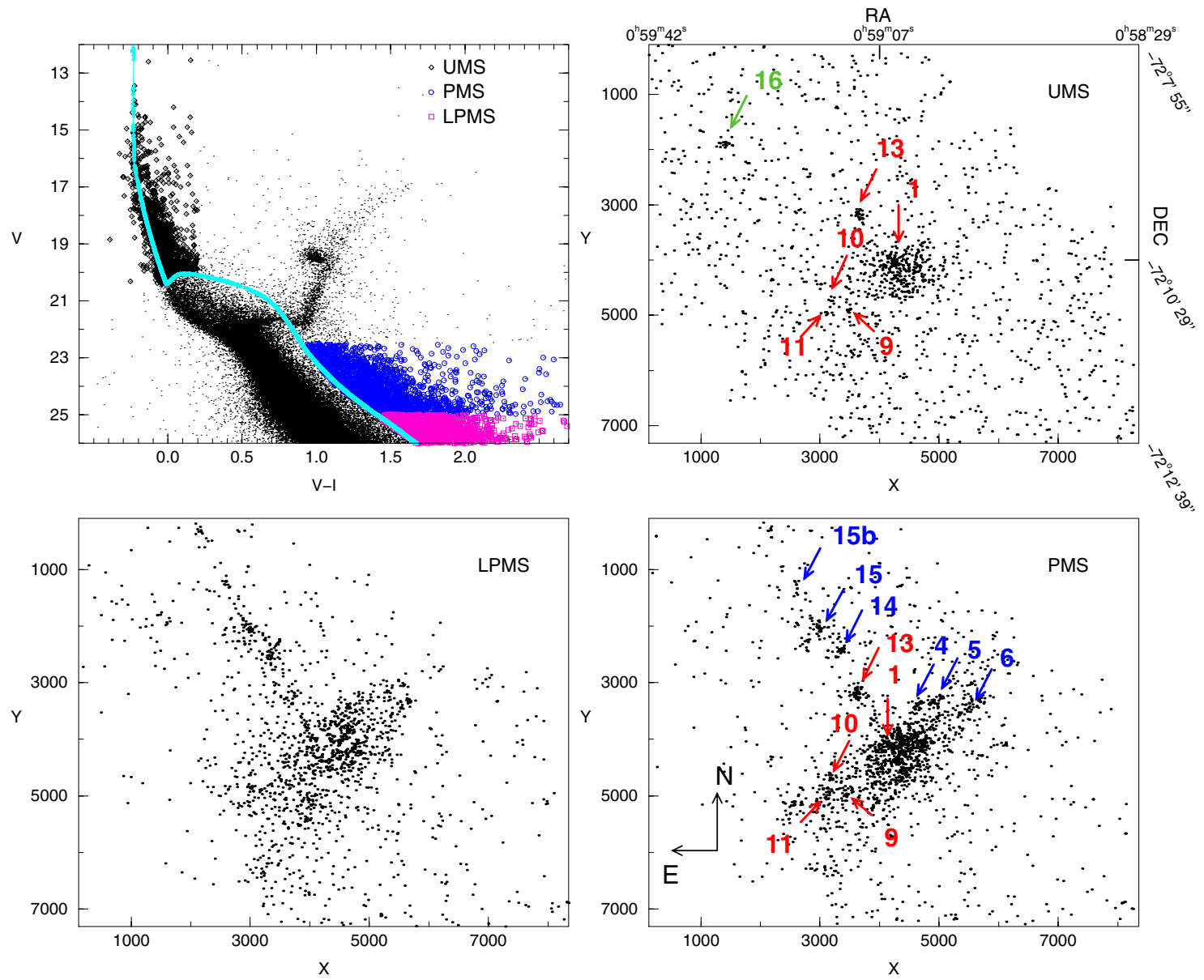

Figure 2. Top left panel: CMD for the region NGC 346. Black open diamonds, blue open circles, and magenta open squares indicate the areas referred to the UMS, PMS, and LPMS stars, respectively. The line corresponds to a 3 Myr isochrone including the PMS phase (Cignoni et al. 2009). The other panels (from top right clockwise) show the corresponding spatial distributions. Red arrows indicate sub-clusters hosting UMS and PMS stars; blue arrows refer to PMS-dominated sub-clusters; and the green arrow refers to the only UMS sub-cluster. The number labeling each sub-cluster follows the nomenclature provided by Sabbi et al. (2007), where the whole catalog with the star coordinates can also be found. In the top right panel, we also show the R.A. and decl. coordinates of the plot extremes and of its center $(X=Y=4000)$.

(A color version of this figure is available in the online journal.)

which host only PMS or UMS stars, respectively. We find that the PMS sub-clusters reside in the northeast part of the Spur (NE-Spur) (SC-14, SC-15, and SC-15b) and in the northwest side of the Bar (NW-Bar) (SC-4, SC-5, and SC-6). However, UMS-PMS sub-clusters are found in the central region (SC-1), in the southern cluster of the Spur (SC-13) and in the southeast side of the Bar (SE-Bar) (SC-9, SC-10, and SC-11). The only sub-cluster to host exclusively UMS stars is SC-16. In fact, SC16 is older than $10 \mathrm{Myr}$ (see, e.g., S07; Cignoni et al. 2010), and its PMS members are too blue to be included in our PMS selection. The situation is radically different for SC-14, SC-15, and SC-15b. Despite the few Myr age of these sub-clusters, there are few, if any, UMS stars: where is the MS counterpart of their observed PMS stars?

To try and understand the sub-cluster similarities and differences, we have divided them in three categories: Group I subclusters are those with a minimum PMS/UMS ratio, Group II sub-clusters are those with the highest PMS/UMS ratio, while we have labeled as Group III the sub-clusters where the ratio was either intermediate or difficult to estimate. Figure 3 shows the clear spatial separation of the three different Groups. Figure 4 shows the CMD for typical Group I sub-clusters (SC-1 and SC-13) and for Group II sub-clusters (SC-14 and SC-15) as



Figure 3. Three boxes superimposed to the NGC346 map identify sub-clusters with similar properties. Solid circles indicate the sub-clusters whose CMDs are discussed in the text. The dashed large circle represents the region of field that has been used to estimate the contamination (see the text).

(A color version of this figure is available in the online journal.) 


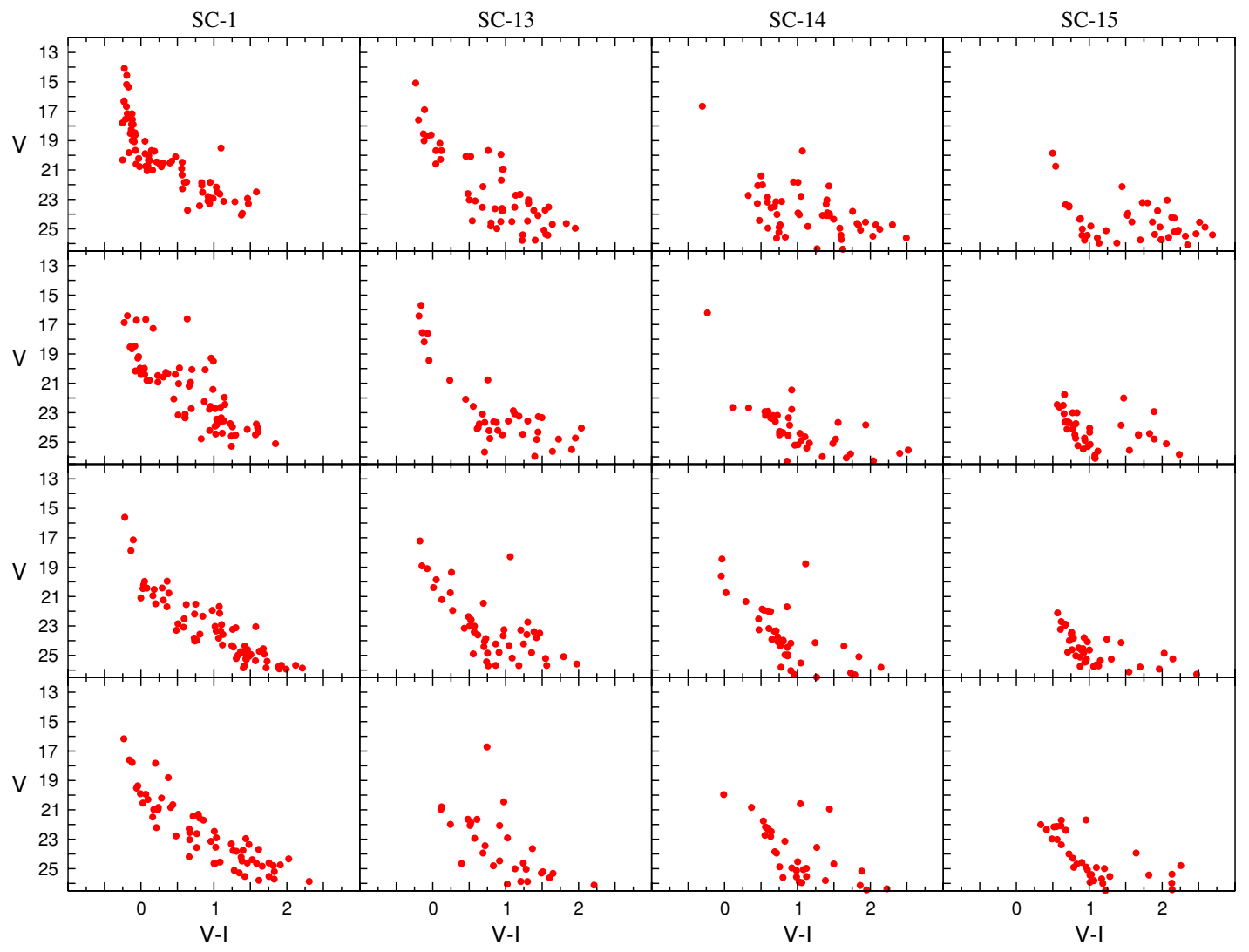

Figure 4. From left to right, the four columns show CMDs for SC-1, SC-13, SC-14, and SC-15, respectively. From top to bottom, stars are shown that belong to progressively more external annuli of equal area centered on the highest density peak. The radius of the inner circle is about 71 pixels $(\approx 1$ pc) for all sub-clusters.

(A color version of this figure is available in the online journal.)

a function of the distance from the sub-cluster center. Different rows refer to stars selected from four equal area annuli.

Excluding the very central region of SC-1, affected by incompleteness, these CMDs trigger the following two interesting questions.

1. Lack of UMS stars. As suggested by the spatial maps, intermediate and massive MS stars seem deficient in subclusters like SC-14. Massive stars are always rare and sub-clusters like SC-14 are tiny pockets of stars; hence, stochastic fluctuations due to small number statistics in the poorly populated high-mass end of the IMF need to be carefully accounted for. Is this sufficient to conclude that the star formation in $\mathrm{SC}-14 / 15$ is a simple scaled-down version of that of SC-1?

2. Redness of PMS stars. The red tail of the PMS distribution extends to redder colors going from SC-1 to SC-15 along the spur. Is their redness intrinsic (age dispersion or circumstellar disks) or caused by obscuring material along the line of sight?

From the analysis of the sub-clusters CMD we find that the Group I members show minimum PMS redness, minimum UMS spread and, by definition, minimum PMS/UMS ratio. The Group II members show the maximum PMS redness and, by definition, the highest PMS/UMS ratio. The Group III members resemble Group I members in relation to the PMS redness and to Group II in relation to the PMS/UMS ratio, while the large color spread among UMS stars is a unique property of this class of sub-clusters.

The next section is dedicated to a quantitative analysis of these groups. In order to reproduce the observed CMD morphology and star counts of the sub-clusters shown in Figure 2 we applied the synthetic CMD approach, combining the $Z=0.004$ Pisa stellar models for PMS stars (see Cignoni et al. 2009) with the same metallicity Padova stellar models (Fagotto et al. 1994) for later evolutionary phases. The explored mass range is between 0.45 and $120 M_{\odot}$. To produce realistic simulations we have incorporated photometric errors and incompleteness corrections as derived from extensive artificial tests on the real images (S07). Different parameters, such as extinction, IMF, binarity, and SFR are varied until the stellar densities and distributions well match the observed CMDs.

To better interpret the morphology of the sub-cluster sequences, we chose sub-cluster radii as the best compromise between the need to bypass the crowding problems, typical of the very inner central regions, and the need to minimize the effect of field star contamination, while still containing a reasonable number of sub-cluster stars. Concerning SC-1, we find that the best strategy is to focus on an annulus around the center from 50 to 282 pixels (see Figure 3). Artificial star tests indicate that in this area stellar detections are more than $90 \%$ complete down to $V \approx 24$. For all minor and satellite sub-clusters, we find that a circle of radius 141 pixels (see Figure 3) allows us to include most of the members with a modest field contamination.

\section{GROUP I}

$$
\text { 4.1. } S C-1
$$

$\mathrm{SC}-1$ is the central and most populous sub-cluster in NGC 346. We have simulated synthetic CMDs based on different combinations of evolutionary (SFH, IMF) and environ- 


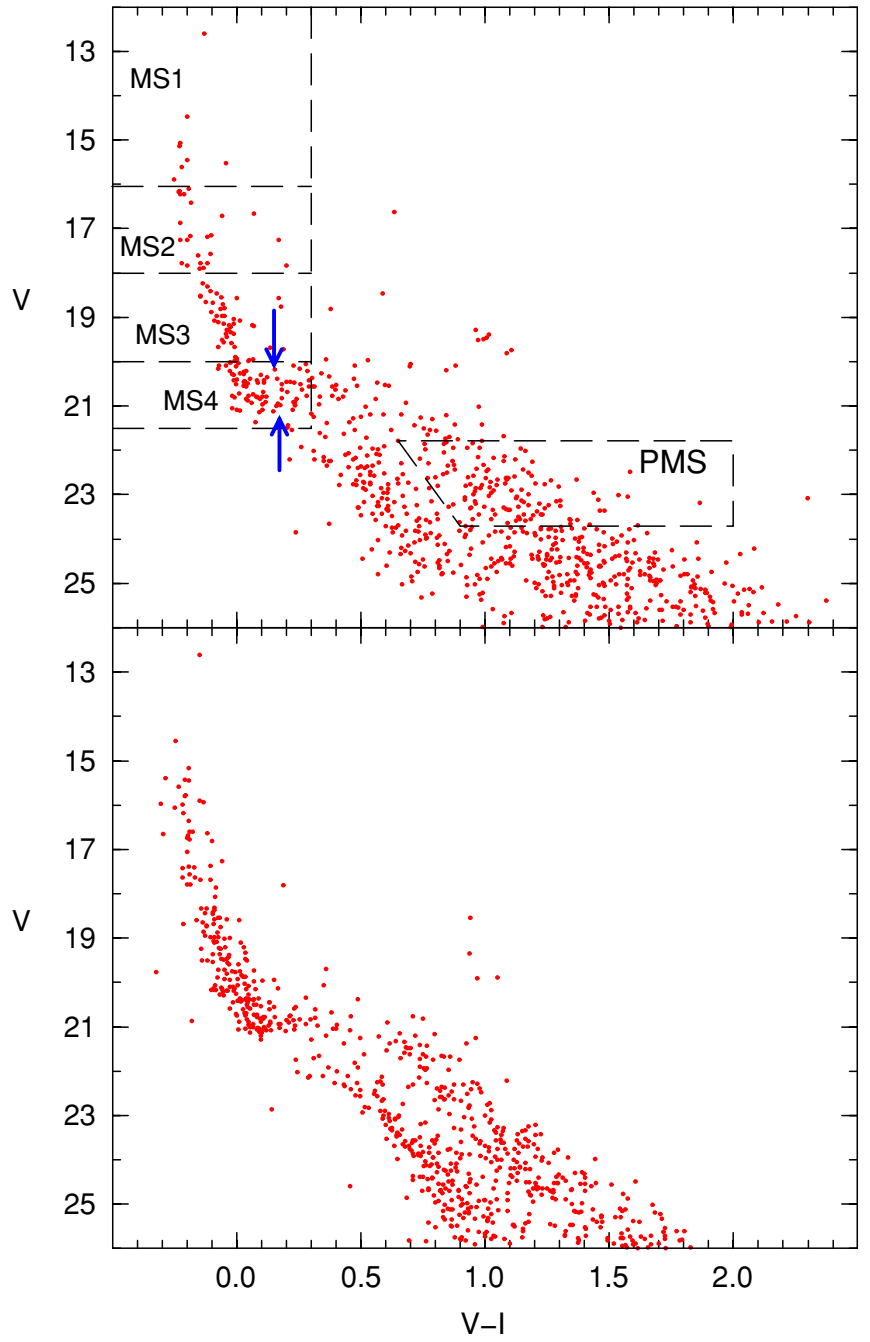

Figure 5. Comparison of observational (top panel) and best fit synthetic CMD (bottom panel) for the sub-cluster SC-1. Also shown are the regions used for characteristic star counts (see the text). There are four such regions along the MS and one for the PMS phase. The theoretical CMDs are calculated with a $30 \%$ of binaries and Salpeter IMF. In the field shown in the figure, the observational and theoretical CMDs contain the same number of stars.

(A color version of this figure is available in the online journal.)

mental parameters (reddening, distance modulus, fraction of binary stars). The best combination of parameters is assessed by examining the star counts in strategic boxes along the main sequence (see labels MS1-MS4 in Figure 5, top panel) and in the PMS. Given the youth of this sub-cluster, likely younger than $10 \mathrm{Myr}$, regions MS1 and MS2 are particularly suitable to constrain the IMF. MS3 and MS4 convey information both on the IMF and on the SFH. The PMS region informs mainly on the IMF (given the uncertainties on the PMS models, the PMS box is used only as a, a posteriori, consistency check). From a numerical point of view, a grid search routine is used to effectively determine the combination of parameters minimizing residuals in these regions. To limit the parameter space, we also considered two additional morphological features: the magnitude $\mathrm{V}$ dispersion of the PMS stars at the TOn (marked with arrows in the top panel of Figure 5) and the number of MS stars in the range of magnitude 21.3 and 21.8 (approximately the 6 Myr TOn and the BS 90 Turn-Off), which is a strong indicator of contamination and/or sub-cluster members older than about 6 Myr. Our solution to handle this contamination was to use as control fields hundred regions of equal area located in the southwest part of the image (see the large dashed circle in Figure 3).

As a first result, a Salpeter IMF (Salpeter 1955), a fraction of binaries of $30 \%$, a foreground reddening $E(B-V)=0.08$, and a distance modulus $(m-M)_{0}=18.9$ provide a good agreement with the data. Concerning the SFH, we find that the stellar production in SC-1 started energetically between 5 and 6 Myr ago, was strong for about 3 Myr (period between 3 and 6 Myr ago), then it dropped, probably victim of feedback from the massive stars of the first generation which quenched the subsequent formation. Before the onset at $6 \mathrm{Myr}$, the observed counts in the CMD window between the 6 Myr TOn and the BS 90 Turn-Off suggest a null or negligible activity: out of nine objects in the magnitude interval $21.3-21.8$ and $V-I<0.45$ at least three can be attributed to the field with a confidence level better than $95 \%$. The residual stars are compatible with a star-forming activity of at most $0.3 \times 10^{-5} M_{\odot} \mathrm{yr}^{-1} \mathrm{pc}^{-2}$ in the period between $8 \mathrm{Myr}$ and $6 \mathrm{Myr}$ ago. During the active phase this region experienced a peak ${ }^{7}$ of about $2 \times 10^{-5} M_{\odot} \mathrm{yr}^{-1} \mathrm{pc}^{-2}$ between 4 and 5 Myr ago, a value much higher than in the center of NGC 602 (see Cignoni et al. 2009), another active star-forming region in the SMC. After this onset, the MS boxes strongly exclude that the star formation was constant or increasing to the present day: according to our best model, only $20 \%$ of the total mass of young stars in the explored region is produced in the last 3 Myr. Moreover, the smoothness of the UMS is not suggestive of any recent short burst.

Figure 5 shows a comparison between our best synthetic CMD (bottom) with the observational one (top). For better visualization, the model has been artificially "contaminated" with field stars taken from an appropriate region of equal area. In broad terms, the UMS and the TOn regions are well reproduced, although the synthetic main sequence appears more fuzzy. Moreover, two discrepancies are noted: (1) our models do not provide satisfactory results about the dispersion of PMS stars, predicting smaller spreads and bluer colors than observed (see also Pozzo et al. 2003; Mayne et al. 2007); and (2) no combination of parameters reproduces the observed number of PMS stars. Once the MS boxes are matched, our best model underestimates it systematically by about $40 \%$. Although part of this difference may arise from our lack of PMS models less massive than $0.45 M_{\odot}$ (potentially present in the PMS box for ages younger than few hundreds of Kyr), a complete solution of the discrepancy seems to require other physical reasons. It is intriguing to note that such discrepancy could easily be resolved by considering an additional episode of star formation younger than $2 \mathrm{Myr}$, accounting for about $500 M_{\odot}$, where the MS phase is artificially suppressed and, therefore, not visible in the CMD. As an alternative, the IMF could be steeper than Salpeter's for masses below $2 M_{\odot}$, thereby creating a larger population of low-mass stars.

In the following, we adopt $\mathrm{SC}-1$ as a reference sub-cluster to compare with minor sub-clusters.

\section{2. $S C-13$}

The SC-13 sub-cluster is less dense than SC-1: excluding MS stars fainter than $V=22$, it accounts for about seven stars per $\mathrm{pc}^{2}$ against about 11 stars per $\mathrm{pc}^{2}$ found in SC-1. Moreover,

\footnotetext{
7 Actually, this is a lower limit, which does not take into account stars below $0.45 M_{\odot}$. When extrapolated using a Salpeter slope down to $0.1 M_{\odot}$, this translates into an upper limit of $3.6 \times 10^{-5} M_{\odot} \mathrm{yr}^{-1} \mathrm{pc}^{-2}$.
} 


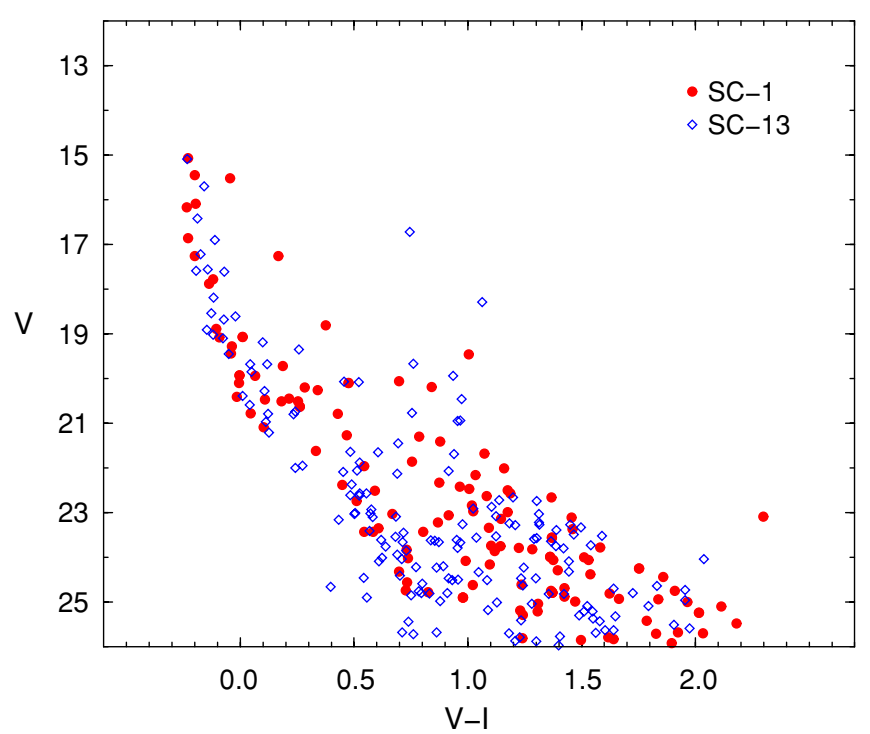

Figure 6. Comparison between SC-1 (filled red circles) and SC-13 (open blue diamonds). The CMD for SC-1 is normalized to the number of SC-13 stars brighter than $V=22$.

(A color version of this figure is available in the online journal.)

Table 1

UMS, PMS, LPMS Star Counts and PMS/UMS Ratio (P/U) for the Labeled Sub-clusters

\begin{tabular}{lccccc}
\hline \hline Group & Sub-cluster & UMS & PMS & LPMS & P/U \\
\hline I & SC-1 & 94 & 195 & 79 & 2.1 \\
& SC-13 & 20 & 35 & 13 & 1.8 \\
& SC-9 & 10 & 19 & 7 & \\
& SC-10 & 7 & 19 & 1 & 2.6 \\
& SC-11 & 7 & 24 & 18 & \\
\hline II & SC-14 & 5 & 23 & 19 & \\
& SC-15 & 0 & 24 & 24 & 10.8 \\
& SC-15b & 0 & 7 & 11 & \\
\hline III & SC-4 & 2 & 22 & 19 & \\
& SC-5 & 2 & 29 & 11 & 10.4 \\
& SC-6 & 3 & 22 & 18 &
\end{tabular}

Notes. All counts are measured inside a radius of 141 pixels, apart SC- 1 that is measured in an annulus around the center between 50 and 282 pixels.

SC-13 is less affected by incompleteness. Despite these differences, when the CMD of SC- 1 is normalized to the number of SC-13 stars brighter than $V=22$ (inside a radius of 141 pixels), the CMD morphologies appear very similar (see Figure 6). First, the color dispersion along the UMS and the TOn magnitude are indistinguishable from one another, suggesting an equivalent star formation duration and onset. Second, the PMS region displays an identical spread and the ratio PMS/UMS is fairly similar (about 2, see Table 1). As already envisaged in Cignoni et al. (2010), the TOn gets brighter (up to $1 \mathrm{mag}$ ) when stars from the inner region of SC-13 (see the top row, second column of Figure 4) are selected. Although numbers are too small to allow statistically significant conclusions, these findings suggest that: (1) the star formation in SC-13 was triggered at the same time of SC-1; (2) the inner region of SC-13 may have experienced a secondary star forming episode about $3 \mathrm{Myr}$ ago. In terms of rate density, we estimate that SC-13 astrated (at peak) about $1.3 \times 10^{-5} M_{\odot} \mathrm{yr}^{-1} \mathrm{pc}^{-2}$.

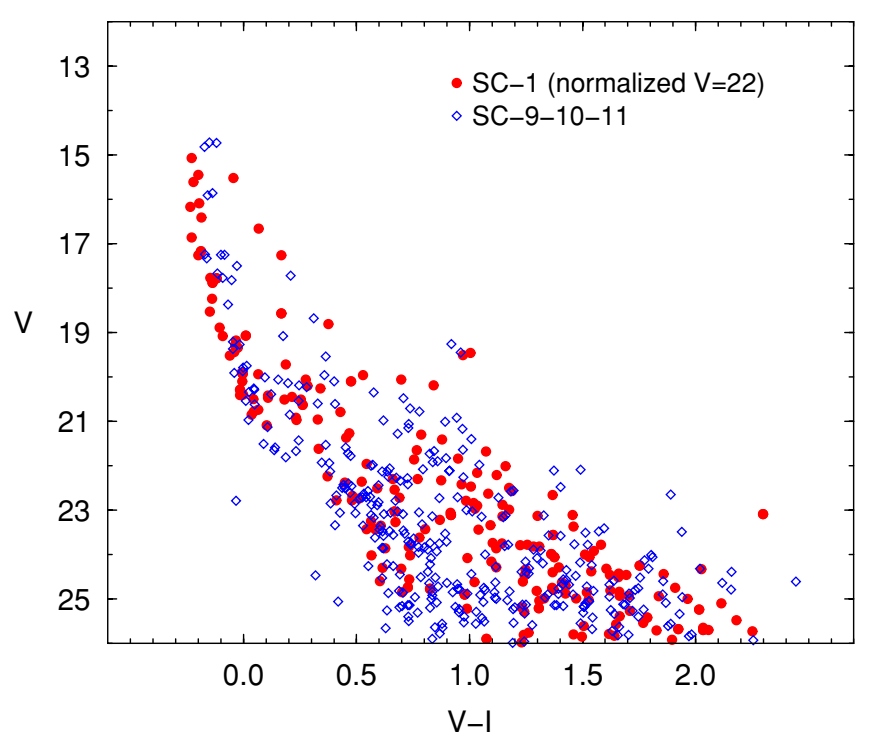

Figure 7. Composite CMD for SC-9, SC-10, and SC-11 (open blue diamonds) superimposed to the CMD for SC-1 (filled red circles) normalized to have the same number of stars brighter than $V=22$.

(A color version of this figure is available in the online journal.)



Figure 8. Comparison between Group II sub-clusters SC-14, 15, 15b (open blue diamonds) and SC-1 (filled red circles) normalized to have the same number of stars brighter than $V=22$.

(A color version of this figure is available in the online journal.)

\section{3. $S C-9, S C-10, S C-11$}

In Figure 7, we present (blue open diamonds) the combination of the CMDs of the sub-clusters SC-9, SC-10, and SC-11, all located in the SE-Bar, superimposed to SC-1 (conveniently normalized to the same number of stars brighter than $V=22$ ). The similarity of morphologies is remarkable: UMS, intermediate mass PMS, and PMS spread overlap. In terms of star counts, the ratio PMS/UMS (about 2.6, see Table 1) is consistent within the expected Poisson fluctuations.

When normalized to the same area, we estimate the maximum SFR density in the SE-Bar to be $0.4 \times 10^{-5} M_{\odot} \mathrm{yr}^{-1} \mathrm{pc}^{-2}$. 


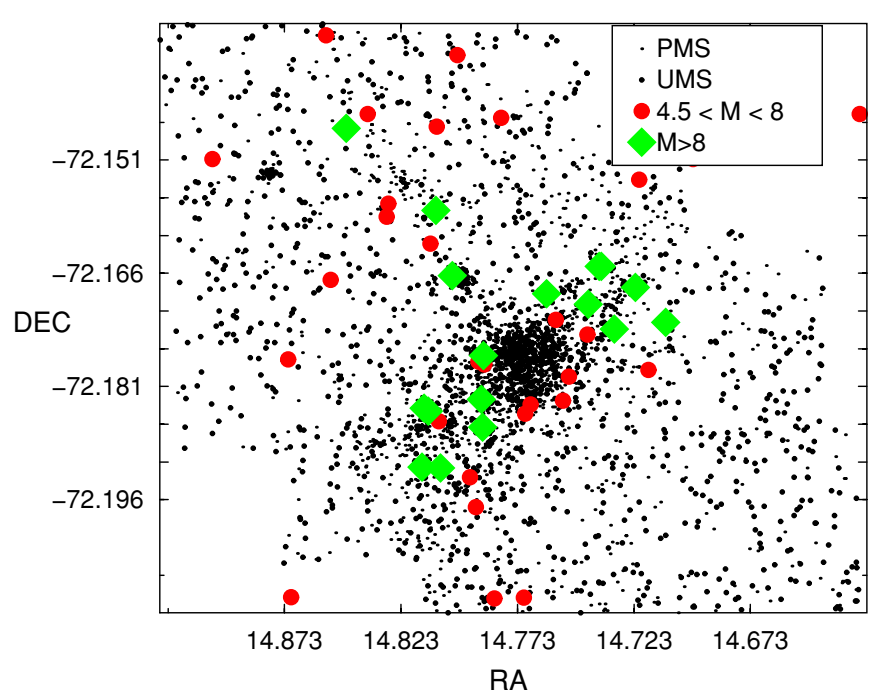

Figure 9. Spatial distribution of the Spitzer sample of intermediate and highmass YSOs (data from Simon et al. 2007) overlaid on the map of PMS (black dots) and UMS stars (large black dots) from Figure 2. Red circles and green diamonds stand for YSOs with estimated mass between 4.5 and $8 M_{\odot}$ and larger than $8 M_{\odot}$, respectively.

(A color version of this figure is available in the online journal.)

\section{GROUP II}

All located in the NE-Spur, the members of this group are embedded in dust and nebulosities. Unlike Group I, these subclusters are mainly composed of low-mass stars with few, if any, UMS stars. This condition strongly hinders any attempt to obtain reliable ages using the TOn. Figure 8 shows a direct comparison between the composite CMD of SC-14, 15, and 15b with SC-1 (re-sampled to have the same number of bright stars with $V<22$ ). Two differences emerge: (1) the Group II CMD shows a ratio of PMS/UMS stars of about 11 (see Table 1), at least a factor of 3 larger than in SC-1, and a lack of intermediate mass PMS stars, which are clearly present in the CMD of SC-1 at $V=20$ and in the color range $0.1<V-I<1$; and (2) in the Group II CMD the LPMS stars are much redder than in $\mathrm{SC}-1$.

Concerning point (1), we notice however that the presence of intermediate mass and massive young stellar objects (YSOs) has been discovered with Spitzer observations by Simon et al. (2007), suggesting that a fraction of UMS stars may exist but be still invisible in optical wavelengths. We show in Figure 9 the distribution of Spitzer YSOs in our field of view.

Point (2) is not caused by either dust between us and the subcluster or diffuse dust within the sub-cluster (otherwise the lower main sequence would also exhibit the color excess) but rather by reddening material intimately related to the individual PMS stars. It is also noteworthy that the UMS is not reddened: either these objects belong to the field or the reddening material affects only the PMS phase (as expected for circumstellar material).

Among the Group II sub-clusters, SC-15 (Figure 10) is the one displaying more significant differences with respect to $\mathrm{SC}-1$. The UMS is definitely underpopulated, with just a couple of stars at best. In addition, the LPMS stars in SC-15 are at least 0.5 mag redder than comparable objects in SC-1, while the main sequence stars fainter than $V=22$ are only slightly redder (0.1 mag). Once again, this would support the idea that reddening material is differentially distributed among PMS stars.

In an attempt to reconcile the PMS samples, we shifted the SC-15 stars along the reddening vector until the color
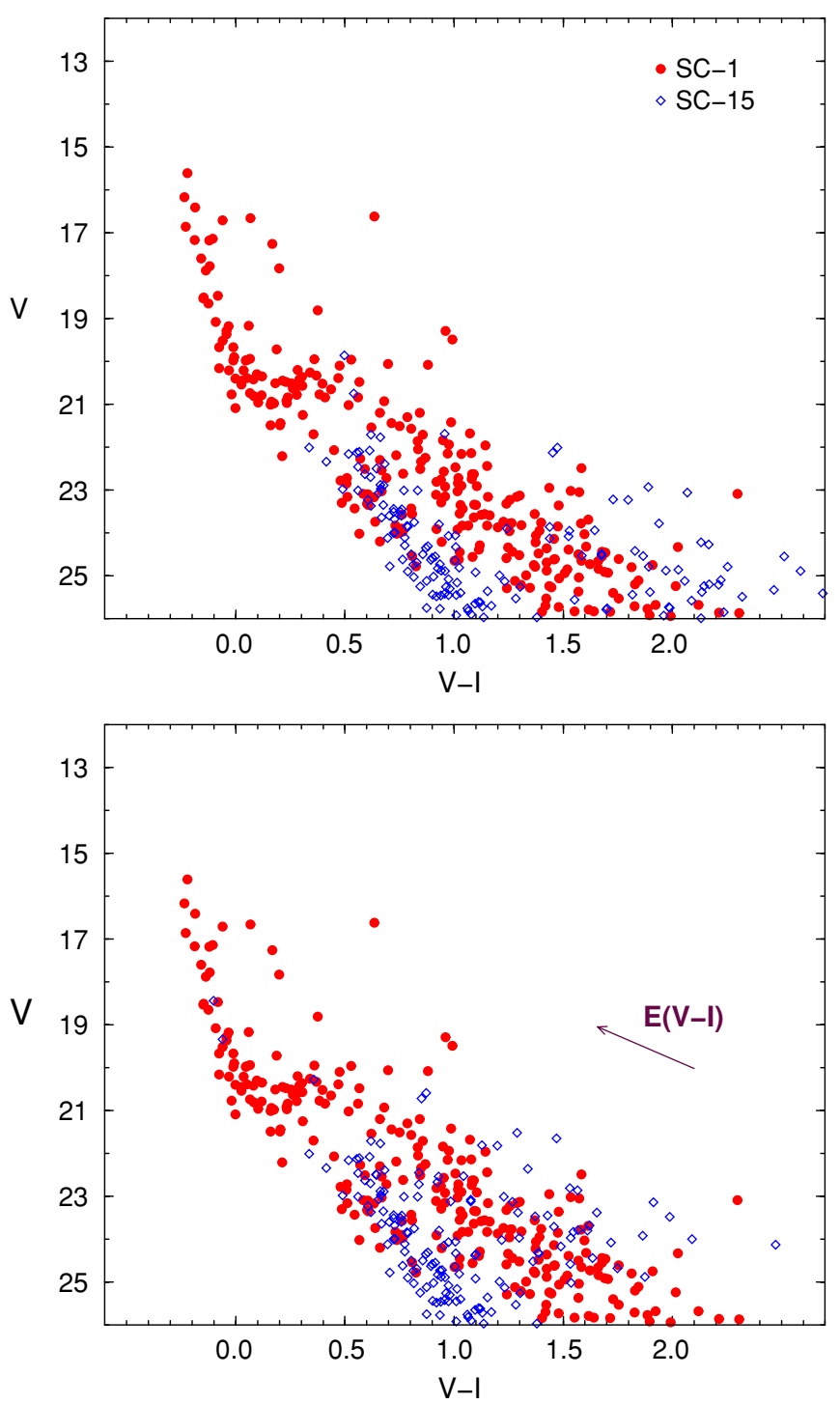

Figure 10. Top panel: comparison between SC-15 (open blue diamonds) and SC-1 (filled red circles) normalized to the same area. Bottom panel: SC-15 after reddening correction applied to PMS stars only.

(A color version of this figure is available in the online journal.)

distribution of PMS in the two sub-clusters CMD overlapped. We derived the optimum shift when the two brighter stars in the SC-15 PMS sample reached the main sequence color. Figure 10 (bottom panel) shows the CMD obtained with this methodology. It is clear that the resulting correction, $E(V-I) \sim 0.6$, is still largely insufficient to reconcile the bulk of PMS stars. Only a differential reddening may overcome the remaining discrepancy. However, the reddening vector (see Figure 10, bottom panel) is almost parallel to the PMS and a full (differential) correction would produce too many bright PMS stars. In other words, although a strong extinction by circumstellar envelopes would be very likely in such young objects, it would necessarily further increase the ratio of PMS stars over UMS stars.

\section{GROUP III}

All members of this group are located in the NW-Bar. Figure 11 shows the combination of CMDs for the sub-clusters SC-4, SC-5, and SC-6 superimposed to the CMD for SC-1. The stellar population in this group shows CMD features which 


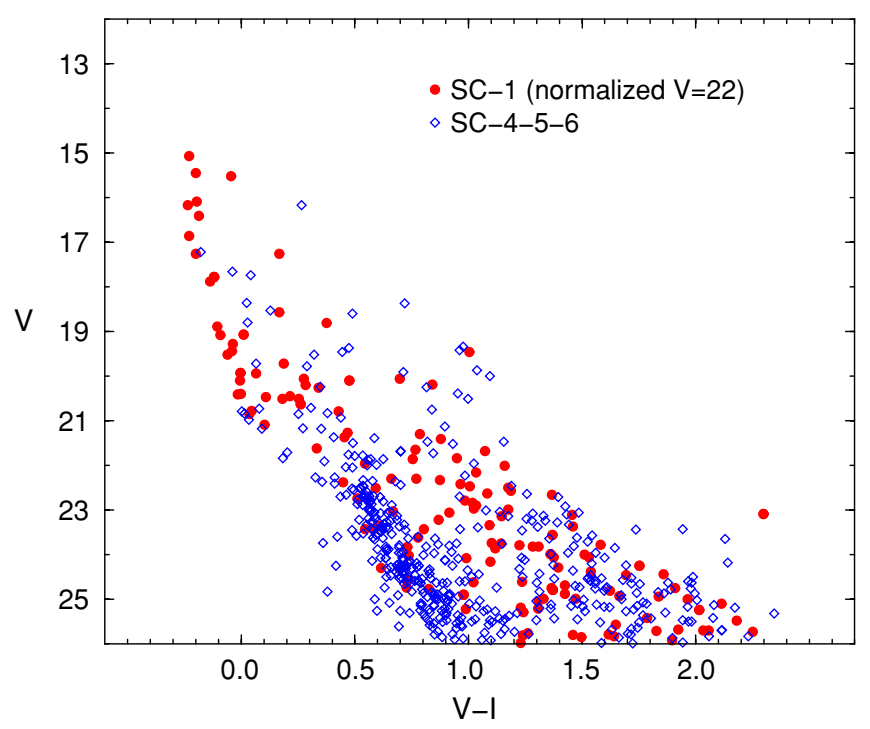

Figure 11. Comparison between the composite CMD for Group III sub-clusters SC-4, SC-5, and SC-6 superimposed to the CMD for SC-1 normalized to have the same number of stars brighter than $V=22$.

(A color version of this figure is available in the online journal.)

are somehow intermediate between Group I and Group II. The color spread in the LPMS is fully consistent with the same sequence in SC-1. However, the ratio PMS/UMS of about 10.4 (see Table 1) is close to the value found in Group II. Moreover, while in SC-1 the UMS is sharply defined and an extended sequence of intermediate mass PMS stars is observed, all bright $(V<23)$ stars in the Group III CMD seem not to lie along any recognizable sequence. Also for these sub-clusters Simon et al. (2007) report the detection of massive embedded YSOs (see Figure 9).

Finally, it is worth noticing that the field contamination, represented by MS stars fainter than $V=22$, is stronger in the NW-Bar and in the NE-Spur than in the SE-Bar because the former regions are closer to the cluster BS 90.

\section{FIELD STAR FORMATION HISTORY}

In addition to a very young population, clearly reflected by clumps or filaments mainly studded by PMS stars, Figure 2 reveals the signatures of an evenly distributed component of UMS stars without an equivalent counterpart of PMS stars. This diffuse and "UMS-dominated" component is clearly inconsistent with a Myr old population and corresponds to a (pervasive) presence of SMC field stars.

In the previous section such field population has been considered only as a mere intruder of the young sub-clusters. Nevertheless, field stars also retain valuable information on the average $\mathrm{SFH}$ in the region. Such history is not represented by either BS 90 or the sub-clusters, since these structures are distinct snapshots in space and time (Gyr old the former, Myr old the latter) of the overall history in the SMC.

We present here a quantitative analysis of the field SFH. To this aim, field stars (red dots in Figure 12) have been chosen as isolated as possible to avoid contamination from either BS 90 or the sub-clusters. However, given the radial profile of BS 90 (see $\mathrm{S} 07$ ), the lower main sequence (fainter than $V \approx 22$ ) as well as the red giant branch (RGB) and the red clump (RC) may still suffer of a residual contamination, potentially leading to an overestimate of the field star formation between 4 and 5 Gyr.

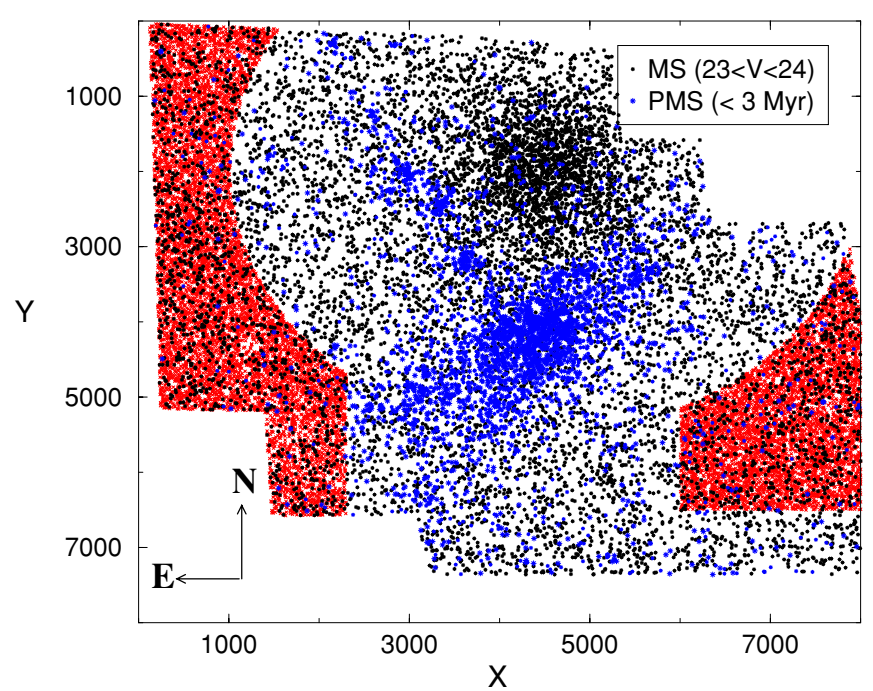

Figure 12. Spatial position of the selected field stars (red dots), PMS stars younger than 3 Myr (blue dots), and main-sequence stars with $23<V<24$ (black dots; see Figure 3 in Cignoni et al. 2010), respectively.

(A color version of this figure is available in the online journal.)

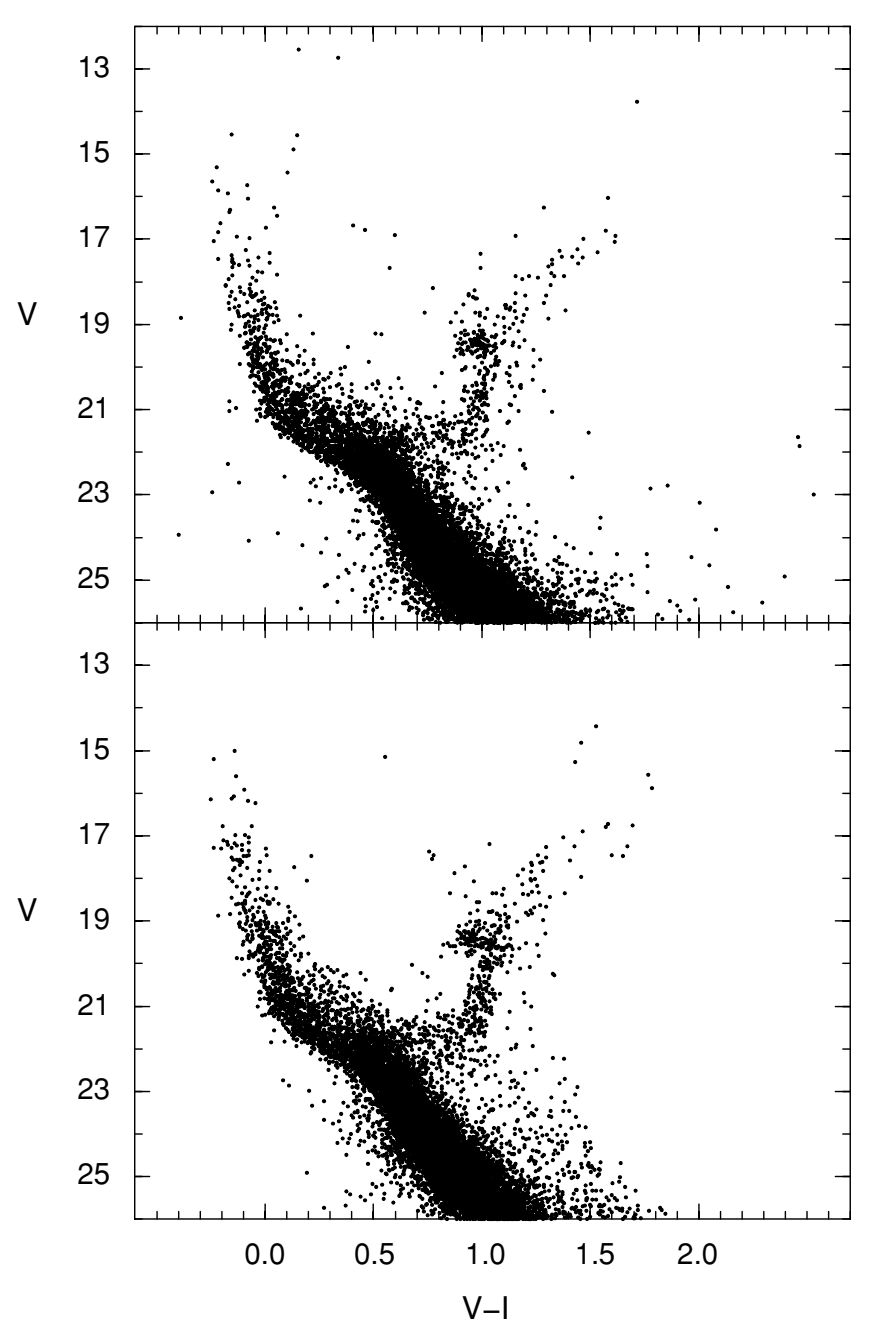

Figure 13. Top panel: CMD for the selected field stars. Bottom panel: best synthetic CMD. 


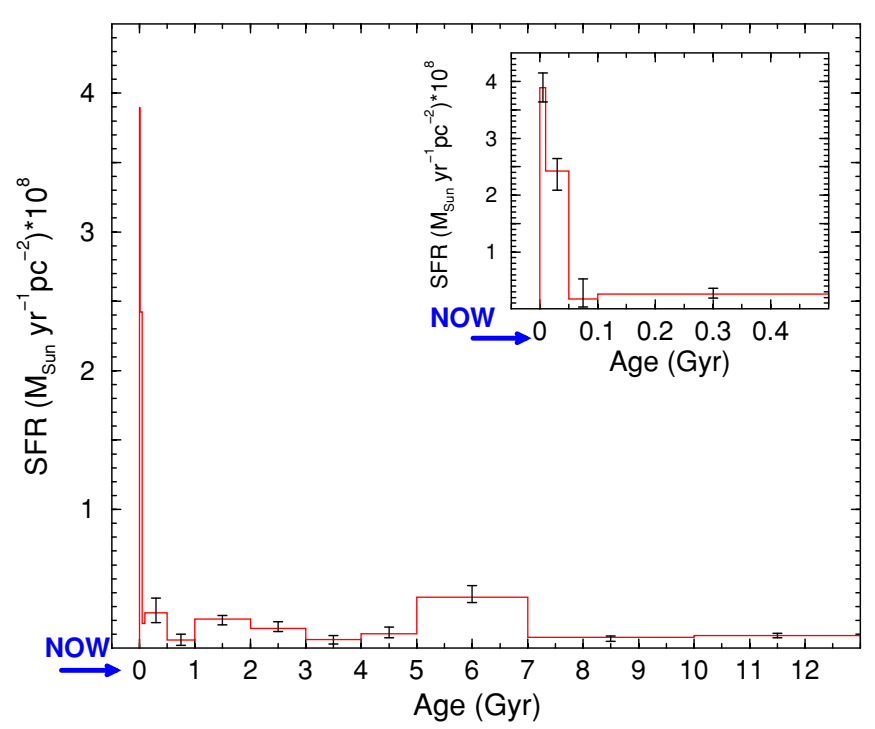

Figure 14. SFH of the SMC field around NGC 346. For the sake of clarity, the most recent $500 \mathrm{Myr}$ are zoomed-in in the upper right onset. The uncertainty on the SFR is also shown for each age bin.

(A color version of this figure is available in the online journal.)

The resulting CMD, containing about 16,000 stars, is shown in the top panel of Figure 13. In order to quantify the corresponding SFH, we compared the observed CMD with an extensive set of simulated histories. In such simulations, the model behavior is changed according to the set of initial parameters, namely, the star formation law and rate $\operatorname{SFR}(t)$, the chemical enrichment law $Z(t)$, the IMF, the fraction of binary stars, the reddening and distance modulus. The comparison between the observed CMD and the model CMDs is done through minimization of the Poissonian $\chi^{2}$ in the number of stars in a set of regions $(0.1 \times 0.1 \mathrm{mag}$ large) of the CMD (cf. Cignoni \& Tosi 2010). In addition, following the approach described, e.g., by Greggio et al. (1998), particular attention has been paid to reproduce the most reliable and evident evolutionary phases, such as the blue loops, the subgiant and the red giant branches, and the main sequence Turn-Offs. To reduce computational time, the SFR is parametrized as a linear combination of fuzzy isochrones with variable duration (partial CMDs). The final uncertainties on the SFH are obtained with a bootstrap technique (see Cignoni et al. 2009 for details).

A morphological comparison between observed and synthetic CMDs allows us to reduce the parameter space. We simulated models with the following metallicity $Z(t)$ (see, e.g., Noël et al. 2009): $Z=0.004$ for stars younger than $2 \mathrm{Gyr}, Z=0.002$ for stars with age between 2 and 5 Gyr, $Z=0.001$ for stars older than 5 Gyr. The adopted IMF has Salpeter's exponent. A $30 \%$ fraction of binaries is assumed. Finally, our synthetic population is corrected for a distance modulus $(m-M)_{0}=18.9$ and a galactic reddening $E(B-V)=0.08$.

The first noticeable result is related to the observed UMS morphology, whose large spread in color is not accounted for by our models either with age or binaries or photometric errors. Even if a differential reddening of about $0.1 \mathrm{mag}$ was effective to solve this discrepancy, the main sequence blue edge in the range $21<V<23$ would be still redder than our models. We suggest that the youngest populations in the field suffer both from differential reddening (which varies by about $0.1 \mathrm{mag}$ ) and from a distance modulus spread of about $0.1 \mathrm{mag}$.

Concerning intermediate to old generations ( $>2 \mathrm{Gyr}$ ), the compactness of the red clump and the thinness of the red giant branch rule out any hypothesis of differential reddening. The magnitude dispersion of the sub-giant branch is consistent with a distance spread of at least $0.2 \mathrm{mag}\left((m-M)_{0}=18.8-19.00\right)$, in good agreement with results obtained for intermediate age star clusters (Glatt et al. 2008). A suggestive scenario may be summarized as follows: (1) the "old" field belongs to an extended halo, evenly distributed around the average distance $(m-M)_{0}=18.9$ and marginally affected by differential reddening; and (2) the "young" field is localized on the farther side of the SMC and suffers differential reddening.

Using these assumptions on distance and reddening, we have proceeded to recover the best combinations of partial CMDs leading to the minimum $\chi^{2}$. The resulting CMD and the corresponding SFH are shown in the bottom panel of Figure 13 and in Figure 14, respectively. The larger errors in the recent activity are mainly due to differential reddening. In terms of star-counts: (1) upper and the lower main sequence (down to $V \approx 25$ ) are well reproduced; (2) the number of red clump stars is always slightly over-predicted, by about 30\%; and (3) blue loop stars are always under-predicted, by 20\%-30\%.

The field SFR is increasing from 13 Gyr ago up to now (see Figure 14). We estimate the average rate density in the last $100 \mathrm{Myr}$ to be $1.4 \times 10^{-8} M_{\odot} \mathrm{yr}^{-1} \mathrm{pc}^{-2}$ $\left(2.5 \times 10^{-8} M_{\odot} \mathrm{yr}^{-1} \mathrm{pc}^{-2}\right.$ when extrapolated using a Salpeter IMF down to $0.1 M_{\odot}$ ) and the average rate density over the whole $13 \mathrm{Gyr}$ lifetime to be $1.5 \times 10^{-9} M_{\odot} \mathrm{yr}^{-1} \mathrm{pc}^{-2}$ $\left(2.7 \times 10^{-9} M_{\odot} \mathrm{yr}^{-1} \mathrm{pc}^{-2}\right.$ when extrapolated). However, about $60 \%\left(38,000 M_{\odot}\right)$ of the stellar mass was assembled in the earliest $8 \mathrm{Gyr}$, with $36 \%\left(21,000 M_{\odot}\right)$ formed between 7 and $5 \mathrm{Gyr}$ ago. For comparison with another SMC star-forming region, NGC 602, in the period between 3 and 13 Gyr ago the field around NGC 346 produced at least six times more mass per $\mathrm{pc}^{2}$ than the field around NGC 602 (Cignoni et al. 2009). Finally, it is also comforting to note that no significant SFR peak in the more recent period 5-4 Gyr ago is found, showing that the contamination from BS 90 is actually minimal.

\section{DISCUSSION AND CONCLUSIONS}

The analysis of the CMDs and of the spatial distribution of the stellar populations in the region of NGC 346 leads to interesting results, both for the clustered and the diffuse components.

Diffuse component. For the diffuse component we find that the majority $(60 \%)$ of the mass astration occurred earlier than $5 \mathrm{Gyr}$ ago, with a peak between 5 and 7 Gyr ago. This is at variance with the suggestion by Harris \& Zaritsky (2004) of a long period of almost no activity from 3 to $8.4 \mathrm{Gyr}$ ago, but in agreement with the SFH of other SMC regions derived by other authors (e.g., Dolphin et al. 2001; McCumber et al. 2005; Cignoni et al. 2009; Noël et al. 2009; Sabbi et al. 2009). We recall that our photometry and those of all the latter authors reach the oldest MS turn-off and therefore a look-back time of 13 Gyr, while the shallower data by Harris \& Zaritsky do not allow the proper description of epochs earlier than a few Gyr.

Over the SMC lifetime the average rate density in the field surrounding NGC 346 is $1.5 \times 10^{-9} M_{\odot} \mathrm{yr}^{-1} \mathrm{pc}^{-2}$. This value is in agreement with those derived by Noël et al. (2009) for several SMC regions. Its SFH is qualitatively similar to that around the other star-forming regions we studied with HST/ACS data, NGC 602 (Cignoni et al. 2009). However, the activity in the NGC 346 region is significantly higher (by a factor of 6) than that in NGC 602. These results are not surprising given the much closer proximity of NGC 346 to the SMC center. The rate of 
star formation in the NGC 346 region (and in NGC 602 as well) appears to have increased in the last tens of Myr from a relatively low and steady regime. We must recall, however, that the SFH recovered at very old epochs is averaged over timescales of Gyr and short episodes of higher activity may remain undetected.

In the last $100 \mathrm{Myr}$ the average SFR density in the field is about $1.4 \times 10^{-8} M_{\odot} \mathrm{yr}^{-1} \mathrm{pc}^{-2}$, a factor of 2-10 higher than in the solar neighborhood (cf. Timmes et al. 1995), 2 orders of magnitude higher than in nearby late-type dwarfs, and similar to the quietest cases of Blue Compact Dwarfs (see Tolstoy et al. 2009 and references therein). In this respect, the SMC region around NGC 346 appears as currently quite active, but we should bear in mind that its current rate might be somewhat overestimated by the possible presence of very young star members of the actual star-forming region itself. For comparison, the peak rate in SC-1 is $2 \times 10^{-5} M_{\odot} \mathrm{yr}^{-1} \mathrm{pc}^{-2}$, 3 orders of magnitude higher than the field average value.

Clustered Component. For the clustered component, we have found that the bulk of the stars in Group I sub-clusters are consistent with a major star-forming episode which started about $6 \mathrm{Myr}$ ago and lasted about $3 \mathrm{Myr}$. After that, their star formation activity has proceeded at a lower rate. As suggested by Palla $\&$ Stahler (2002) for the stellar group Taurus-Auriga, massive stars, dispersing the gas that would have been part of the new generations, may be the culprit for such a "deceleration" in the most recent star-forming activity.

Our models do not fully explain the large color spread shown by PMS stars and the large observed number of PMS stars. The PMS indeed shows a redward broadening which is not reproduced by either our youngest isochrones or photometric scatter. Hennekemper et al. (2008) suggested that a combination of differential reddening, variability, and binarity may account for the observed spread. We consider variable reddening affecting individual PMS stars a more likely explanation (also taking into account that our PMS evolutionary tracks do not include circumstellar reddening). Moreover, our models seem to underestimate the observed PMS counts by about $40 \%$ (possibly and in part because our PMS tracks do not cover masses smaller than $0.45 M_{\odot}$ ). We speculate that a very young generation of stars, so young as not to have had time to assemble more massive stars, could account both for the observed excess of PMS stars and for the intrinsic redness of such stars; otherwise, the IMF must be steeper than Salpeter.

While the origin of these discrepancies is still unclear, the comparison with CMDs of stars in different locations provides clues to complete the puzzle.

Low-mass PMS stars dominate the star counts among Group II sub-clusters, showing a ratio of PMS/UMS stars that is at least three times higher than in Group I. Such a population should have an MS counterpart, which is however not visible. Among all the sub-clusters in NGC 346, those in Group II host the reddest PMS stars. Only a strong and differential reddening correction could reconcile the Group II PMS with the SC-1 PMS, but it would pay the price of exacerbating the lack of UMS stars. Vice versa, an age effect, with the Group II sub-clusters experiencing now their first episode of star formation, may explain both the PMS redness and the higher PMS/UMS ratio. If the NE-Spur, where the Group II sub-clusters are located, is indeed the youngest region of NGC 346, a fraction of stars may have managed to retain the circumstellar material and appear redder. Moreover, the youth of the system and the peculiar distribution of NE-Spur stars, preferentially located along filaments, may determine the paucity of UMS. In this case the peak gas den- sities in the filaments would be insufficient to produce massive stars, such as those detected in SC-1. As a consequence, the ratio of low-mass to massive stars is higher where the density of massive stars is lower.

This trend is also noted by Panagia et al. (2000) in the field of SN1987A. It indicates that star formation processes for different ranges of stellar masses are rather different and/or require different initial conditions. An interesting corollary may be that, if the youngest objects have a filamentary distribution (where presumably the gas density was higher), a critical density threshold exists below which the star formation is suppressed. The reason for this is probably related to the pristine conditions in the parental molecular clouds, like temperature or turbulence.

As an alternative to environmental effects, the lack of UMS stars may reflect the build up timescales of different masses. More massive stars may form later than low-mass stars because they need more time to collect enough material to start the formation. In this case, the Group II sub-clusters are simply too young to have produced massive stars.

The analysis of Hennekemper et al. (2008) and Gouliermis et al. (2008) provides independent support to the age hypothesis. Fitting Seiss isochrones, Hennekemper et al. (2008) derived for the NE-Spur sub-clusters ages younger than 2.5 Myr, while Gouliermis et al. (2008) concluded that the star formation there was recently triggered (see Figure 1 in Gouliermis et al. 2008) by the massive progenitor of SNR B005-7724. We notice however that Nazé et al. (2002) argued that the SNR should be located in front of the luminous blue variable HD-5980 and not really within the NGC 346 region.

From the point of view of the gas, the NE-Spur is a natural place to find new stellar generations. Spectroscopic observations of carbon monoxide prove the existence of reservoirs of cold molecular gas in the spatial region around SC-14 and SC-15 (see Rubio et al. 2000) while the distribution of the radiation field at $160 \mathrm{~nm}$ is clearly confined to the Bar, and it is not detected in the Spur (except in SC-13). In other words, the extreme NESpur contains residual reservoirs of molecular gas that may be fueling the star formation.

The Group III sub-clusters exhibit (1) the same anomalous ratio PMS/UMS stars found in Group II (hence at variance with Group I); (2) a color spread and redness among LPMS that is perfectly in line with what is found in Group I (hence at variance with Group II); and (3) a color spread among UMS that is at variance both with Group I and with Group II members.

This suggests a picture where the Group III sub-clusters are in an intermediate state between those in Group II and those in Group I. If the underlying parameter is the age, these sub-clusters are sufficiently old that low-mass PMS stars have already completed their accretion phase (hence their color dispersion is consistent with the "evolved" cluster SC-1), but at the same time so young that intermediate and massive stars are still approaching the UMS. As for the NE-Spur, another key to interpretation is the available gas out of which the presently observable stars were assembled. In this picture, the gas density in the NW-Bar was such that intermediate and massive stars were formed more slowly than in the SE-Spur.

These findings appear to support the view that low-mass stars form more "easily" than massive ones either because they need less gas density or lower rates of accretion. Observational support for this was already presented, e.g., by Ruppert \& Zinnecker (2009). Likewise, the Spitzer detection (Simon et al. 2007) of proto-OB stars in Group II and Group III sub-clusters may explain the apparent lack of UMS stars in terms of age. 
As comprehensively reviewed by Zinnecker \& Yorke (2007) no consensus has been reached yet on which is the most likely process for massive star formation: monolithic collapse in isolated cores, competitive accretion in a protocluster environment or stellar collision, and mergers in very dense systems. NGC 346 seems to favor one of the latter two (or both) but we should wait for further high-resolution observations at longer wavelengths (e.g., with WFC3 on board of HST, but also ALMA and James Webb Space Telescope, JWST, and eventually with Extremely Large Telescopes (ELTs)) to get a better insight into its star formation process based also on its still embedded, not visible, youngest objects.

In the conditions described above an assessment of the IMF in the NGC 346 region is risky. There is no doubt that in several sub-clusters the number of existing massive stars is definitely lower than predicted by a Salpeter IMF. Sabbi et al. (2008) already pointed out that in the region massive stars are underrepresented. We speculate that the paucity of UMS in NGC 346 has a double origin: the maximum mass populating Group II sub-clusters is a consequence of the radiation feedback, while the maximum mass populating the PMS sub-clusters is mainly a matter of youth. From a general point of view, our result goes in the same direction of a relation between the mass of the most-massive star in the cluster and the mass of its parent star cluster as suggested by Weidner \& Kroupa (2006). On the other hand, if indeed clusters form in an ordered fashion producing first low-mass stars and then proceeding to assemble more massive stars till a maximum mass is born whose feedback halts the collapse, how is it possible to explain the existence of sub-clusters hosting only PMS stars? We expect ALMA, Herschel, and JWST to provide the necessary clues.

M.C. and M.T. acknowledge financial support through contracts ASI-INAF-I/016/07/0 and PRIN-MIUR-2007JJC53X001. Partial support for U.S. research in program GO10248 was provided by NASA through a grant from the Space Telescope Science Institute, which is operated by the Association of Universities for Research in Astronomy, Inc., under NASA contract NAS 5-26555.

\section{REFERENCES}

Bica, E. L. D., \& Schmitt, H. R. 1995, ApJS, 101, 41

Cignoni, M., Degl'Innocenti, S., Prada Moroni, P. G., \& Shore, S. N. 2006, A\&A, 459, 783

Cignoni, M., \& Tosi, M. 2010, Adv. Astron., 2010, 158568

Cignoni, M., Tosi, M., Sabbi, E., Nota, A., Degl'Innocenti, S., Prada Moroni, P. G., \& Gallagher, J. S. 2010, ApJ, 712, L63

Cignoni, M., et al. 2009, AJ, 137, 3668

Contursi, A., et al. 2000, A\&A, 362, 310

Dolphin, A. E., Walker, A. R., Hodge, P. W., Mateo, M., Olszewski, E. W., Schommer, R. A., \& Suntzeff, N. B. 2001, ApJ, 562, 303

Fagotto, F., Bressan, A., Bertelli, G., \& Chiosi, C. 1994, A\&AS, 105, 29

Glatt, K., et al. 2008, AJ, 136, 1703

Gouliermis, D. A., Chu, Y.-H., Henning, T., Brandner, W., Gruendl, R. A., Hennekemper, E., \& Hormuth, F. 2008, ApJ, 688, 1050

Greggio, L., Tosi, M., Clampin, M., de Marchi, G., Leitherer, C., Nota, A., \& Sirianni, M. 1998, ApJ, 504, 725

Harris, J., \& Zaritsky, D. 2004, AJ, 127, 1531

Hennekemper, E., Gouliermis, D. A., Henning, T., Brandner, W., \& Dolphin, A. E. 2008, ApJ, 672, 914

Mayne, N. J., Naylor, T., Littlefair, S. P., Saunders, E. S., \& Jeffries, R. D. 2007, MNRAS, 375, 1220

McCumber, M. P., Garnett, D. R., \& Dufour, R. J. 2005, AJ, 130, 1083

Nazé, Y., Hartwell, J. M., Stevens, I. R., Corcoran, M. F., Chu, Y.-H., Koenigsberger, G., Moffat, A. F. J., \& Niemela, V. S. 2002, ApJ, 580, 225

Noël, N. E. D., Aparicio, A., Gallart, C., Hidalgo, S. L., Costa, E., \& Méndez, R. A. 2009, ApJ, 705, 1260

Nota, A., et al. 2006, ApJ, 640, L29

Palla, F., \& Stahler, S. W. 2002, ApJ, 581, 1194

Panagia, N., Romaniello, M., Scuderi, S., \& Kirshner, R. P. 2000, ApJ, 539, 197

Pozzo, M., Naylor, T., Jeffries, R. D., \& Drew, J. E. 2003, MNRAS, 341, 805

Rochau, B., Gouliermis, D. A., Brandner, W., Dolphin, A. E., \& Henning, T. 2007, ApJ, 664, 322

Rubio, M., Contursi, A., Lequeux, J., Probst, R., Barbá, R., Boulanger, F., Cesarsky, D., \& Maoli, R. 2000, A\&A, 359, 1139

Ruppert, J., \& Zinnecker, H. 2009, Astron. Nachr., 330, 773

Sabbi, E., et al. 2007, AJ, 133, 44

Sabbi, E., et al. 2008, AJ, 135, 173

Sabbi, E., et al. 2009, ApJ, 703, 721

Salpeter, E. E. 1955, ApJ, 121, 161

Schmeja, S., Gouliermis, D. A., \& Klessen, R. S. 2009, ApJ, 694, 367

Simon, J. D., et al. 2007, ApJ, 669, 327

Timmes, F. X., Woosley, S. E., \& Weaver, T. A. 1995, ApJS, 98, 617

Tolstoy, E., Hill, V., \& Tosi, M. 2009, ARA\&A, 47, 371

Tosi, M., Greggio, L., Marconi, G., \& Focardi, P. 1991, AJ, 102, 951

Weidner, C., \& Kroupa, P. 2006, MNRAS, 365, 1333

Zinnecker, H., \& Yorke, H. W. 2007, ARA\&A, 45, 481 